\title{
A List of the Jews (313 Persons) who Arrived in Palestine from Romania (May 24, 1944)
}

\section{Emanuel B* \\ School of socio-human sciences, University of Suceava, Romania}

*Corresponding author: Emanuel Bălan, School of socio-human sciences, University of Suceava, Suceava county, Romania, Tel: 86 027- 68764004; Fax: 86 027- 68764004; Email: emanuel.balan_tgn@yahoo.com

\section{Research article}

Volume 3 Issue 3

Received Date: August 10, 2020

Published Date: August 27, 2020

DOI: $10.23880 /$ phij-16000147

\section{Abstract}

From the first days of the Antonescu regime, the Jews chose the path of Palestine. In November 1940, three ships with over 3,500 Jews left the port of Tulcea for Palestine. In 1941 only one ship left Romania with 789 Jews. Most emigrations took place in 1942 and 1944, obviously with the consent of the Romanian authorities.

Keyword: Alia; Jews; Emigration; Romania; Second World War; Palestine

\section{Introduction}

Emigration to Israel from Romania (Alia) during the Second World War also experienced tragic moments. On February 24, 1942, the greatest tragedy in the history of the Alia movement took place, the sinking of the ship Struma in the Black Sea, near the port of Istanbul. The ship that left Contanța port on December 12, 1941 had on board 764 emigrants crowded in inhumane conditions. Following the torpedoing of the ship by a Russian submarine, only one person managed to survive1.

Despite this tragedy, Alia continued, even though the road was littered with numerous obstacles and dangers. Unfortunately, on August 5, 1944, a second tragedy occurred, the ship Mefkure, with 389 emigrants, was torpedoed by a Soviet submarine, surviving only five people ${ }^{2}$.

During 1942, from the ports of Constanța, Sulina, Tulcea and Brăila, eight ships left for Israel, with on board approximately 250 Jewish emigrants: Dor de Val (14

1 Haim Barles, Acțiunile de salvare din perioada Holocaustului, Tel Aviv, 1975, pp. 178-181, 234-235

2 Iaacov Geller, Tragedia scufundării vasului Mefkure, august 1944, în „Holocaust”, vol. 19, 1991, Tel Aviv, pp. 148-153 emigrants); Eouxine (11 emigrants); Mihai (13 emigrants); Mircea (40 emigrants); Crai Nou (12 emigrants); Europe (20 emigrants); Dora (15 emigrants) and Viitorul (120 emigrants). During 1944, from March to August, nine ships left the port of Constanța, with a total of 3419 emigrants, of which only 3024 reached the shores of Israel. The ships that left Constanța were: Milka A (239 emigrants); Marița A (224 emigrants); Balacitta (273 emigrants); Milka B (517 emigrants); Marița B (318 emigrants); Kazbek (735 emigrants); Bulbul (410 emigrants); Morina (308 emigrants) and Mekfure (389 emigrants) ${ }^{3}$.

In this study we will focus on the list of the Jews who arrived in Palestine from Romania, on May 24, 1944. The ship, a motor sailboat under the Bulgarian flag, was called the Milka. Under the command of the bulgarian Hristo Lazarof, he set sail from the port of Constanța, on April 27,1944 , in perfect airworthiness, the voyage proceeding without incident $t^{4}$. In the following we will present the list of imigrants who arrived in Palestine from Romania on the day of may 24, 1944. Of the 313 jews who arrived in Palestine

3 Mordehai Naor, Emigrarea 1934-1948, Tel Aviv, 1981, pp. 141-143.

4 Koslinski N, Stănescu R, Marina Română în al doilea război mondial (1939-1945), vol. IIII (1944-1945), București, Editura Modelism Internațional, 1998, p. 46. 


\section{Philosophy International Journal}

from Romania, 50 were children under 18 and 130 were women $^{5}$.

5 ACSIER fond III, dosar 361, ff. 120-124.

\begin{tabular}{|c|c|c|c|c|}
\hline No. & Name \& Surname & Age & Fathers name & Place of residence \\
\hline 1 & Ajchenazy Anazel & 11 & Nuchim & Hotin \\
\hline 2 & Ajchenazy Jochevet & 7 & Nuchim & Hotin \\
\hline 3 & Almosnino Jean & 35 & Samuel & Bucharest \\
\hline 4 & Almosnino Radicz & 28 & Albert & Bucharest \\
\hline 5 & Alter Smil & 21 & Jacob & Bucharest \\
\hline 6 & Arie Ulrich & 33 & Isidor & Bucharest \\
\hline 7 & Arie Eva & 31 & David & Bucharest \\
\hline 8 & Arie Davy & 5 & Ulrich & Bucharest \\
\hline 9 & Aschkenasi Heinrich & 37 & Lasar & Budapest \\
\hline 10 & Aschkenasi Scheindel & 26 & David & Budapest \\
\hline 11 & Avram Silvy & 19 & Avram & Iași \\
\hline 12 & Avram Arnold & 16 & Avram & Iași \\
\hline 13 & Avram David & 39 & Naftali & Bucharest \\
\hline 14 & Bacal Jean & 46 & Uscher & Bucharest \\
\hline 15 & Bacal Grina & 26 & Marcu & Bucharest \\
\hline 16 & Barbasch Tirla & 19 & Leib & Iași \\
\hline 17 & Barghida Margaretta & 19 & Josef & Arad \\
\hline 18 & Bartha Ladislau & 48 & Simon & Turda \\
\hline 19 & Bartha Paraschiva & 43 & Leopold & Turda \\
\hline 20 & Barry Zailig & 45 & Haim & Ploiești \\
\hline 21 & Barry Vica & 25 & Mosche & Ploiești \\
\hline 22 & Barry Miriam & 8 & Zailig & Ploiești \\
\hline 23 & Basch Frida & 21 & Jacob & Bucharest \\
\hline 24 & Baumel Matei & 28 & Naftali & Bucharest \\
\hline 25 & Bercovici Berta & 58 & Avram & Bucharest \\
\hline 26 & Bertheil Siloya & 24 & Freidrich & Bucharest \\
\hline 27 & Birnbaum Z. Osias & 20 & Mates & Bucharest \\
\hline 28 & Blovincic Solomon & 54 & Israel & Bucharest \\
\hline 29 & Blovincic Sofie & 54 & Mosche & Bucharest \\
\hline 30 & Brandman Zisu & 19 & Israel & Iași \\
\hline 31 & Brauvels Perl & 26 & Iacov & Iași \\
\hline 32 & Burstein Rosa & 22 & Marcu & Iași \\
\hline 33 & Burstein Josef & 23 & Itzic & Iași \\
\hline 34 & Chelpner Barcu & 44 & Sloime & Iași \\
\hline 35 & Chelpner Ruhla Lea & 41 & Ithac & Iași \\
\hline 36 & Cohn Ignatz & 21 & Max & Brașov \\
\hline
\end{tabular}




\begin{tabular}{|c|c|c|c|c|}
\hline 37 & Croitru Lora & 22 & Sloima & Piatra-Neamț \\
\hline 38 & Damideanu Adolf & 19 & Strul & Iași \\
\hline 39 & Dauber Sabina & 23 & Mordco & Transnistria \\
\hline 40 & Diamant Elemer & 43 & Iuliu & Brașov \\
\hline 41 & Diamant Elisabeta & 36 & Jacob & Brașov \\
\hline 42 & Diamant Richard & 16 & Elemer & Brașov \\
\hline 43 & Diamant Iosna & 14 & Elemer & Brașov \\
\hline 44 & Diamant Toama & 5 & Elemer & Brașov \\
\hline 45 & Echkhaus Bernard & 42 & Chidalic & Bucharest \\
\hline 46 & Echkhaus Pepi & 30 & Meier & Bucharest \\
\hline 47 & Echkhaus Edith & 7 & Bernard & Bucharest \\
\hline 48 & Echkhaus Chidale & 72 & Maischa & Bucharest \\
\hline 49 & Eisenberg Avram & 50 & Israel & Bucharest \\
\hline 50 & Eisenberg Ester Dora & 38 & Ichiel & Bucharest \\
\hline 51 & Eisenberg Sergiu & 12 & Avram & Bucharest \\
\hline 52 & Eisenberg Belia & 10 & Avram & Bucharest \\
\hline 53 & Eisengratz Marietta & 19 & Izhac & Bacău \\
\hline 54 & Eisenntadter Nicolas & 30 & Filip & Bucharest \\
\hline 55 & Eisenntadter Eleonore & 35 & Karl & Bucharest \\
\hline 56 & Elias Martha & 17 & David & Timișoara \\
\hline 57 & Eliovici Adalbert & 20 & Josef & Sibiu \\
\hline 58 & Engler Moses & 35 & David & Cernăuți \\
\hline 59 & Epstein Jancu & 20 & Sama & Iași \\
\hline 60 & Fagadau Littman David & 42 & Herșcu & Bucharest \\
\hline 61 & Fagadau Littman Lea & 33 & Haim & Bucharest \\
\hline 62 & Fagadau Littman Rudy & 14 & David & Bucharest \\
\hline 63 & Feldmann Filip & 30 & Naumann & Bucharest \\
\hline 64 & Feldmann Bitzi & 20 & Aurel & Bucharest \\
\hline 65 & Feller Hersch & 43 & Haim & Bucharest \\
\hline 66 & Feller Ella & 37 & Josef & Bucharest \\
\hline 67 & Feller Dalia & 11 & Hersch & Bucharest \\
\hline 68 & Finkelstein Froim & 25 & Moritz & Focșani \\
\hline 69 & Fischel Moritz & 19 & Fridrich & Bucharest \\
\hline 70 & Fischman Mihai & 20 & Jacob & Arad \\
\hline 71 & Forst Sonia & 19 & Melic & Iași \\
\hline 72 & Frankel Ignatz & 23 & Cerman & Cernăuți \\
\hline 73 & Furman Josub & 22 & Isac & Bucharest \\
\hline 74 & Gelperin Leon Isaac & 23 & David & Bucharest \\
\hline 75 & Gelber Izchak & 22 & Hersch & Bucharest \\
\hline 76 & Goldenberg Mosche & 60 & Saul & Bucharest \\
\hline
\end{tabular}




\begin{tabular}{|c|c|c|c|c|}
\hline 77 & Goldenberg Rachel & 55 & Hans & Bucharest \\
\hline 78 & Goldstein Sirena & 18 & Solomon & Timișoara \\
\hline 79 & Goltz Palias & 23 & Jzak & Bucharest \\
\hline 80 & Gora Francisco & 30 & - & Budapest \\
\hline 81 & Gottesman Erich & 23 & Strul & Cernăuți \\
\hline 82 & Grinberg Ghedalia & 30 & Simon & Focșani \\
\hline 83 & Grinberg Jocheved & 15 & Mendel & Iași \\
\hline 84 & Grunberg Noe & 45 & Marcu & Bucharest \\
\hline 85 & Grunberg Manuela & 38 & Moritz & Bucharest \\
\hline 86 & Grunberg Israel & 21 & Burach & Bacău \\
\hline 87 & Grunberg Ernestine & 22 & Alter & Iași \\
\hline 88 & Grunstein Roza & 16 & Julius & Iași \\
\hline 89 & Gurfain Jacob & 23 & Avram & Budapest \\
\hline 90 & Handelmann Itzchak & 19 & Elieser & Iași \\
\hline 91 & Harband Artu & 35 & Solomon & Cernăuți \\
\hline 92 & Harband Chitel & 30 & Leib & Cernăuți \\
\hline 93 & Harscovici Rubin & 43 & David & Bucharest \\
\hline 94 & Harscovici Sara & 30 & Avram & Bucharest \\
\hline 95 & Harscovici Doral & 7 & Rubin & Bucharest \\
\hline 96 & Hasenfratz Nisu & 21 & Meier & Iași \\
\hline 97 & Heittner Israel Dr. Rab. & 37 & Itzak Meir & Bârlad \\
\hline 98 & Heittner Tamara & 36 & Meschulom & Bârlad \\
\hline 99 & Heittner Tipora & 7 & Israel & Bârlad \\
\hline 100 & Heittner Itzhac Mair & 3 & Israel & Bârlad \\
\hline 101 & Held Eleonora & 33 & Isidor & Cernăuți \\
\hline 102 & Held Calman & 8 & Gustav & Cernăuți \\
\hline 103 & Held Sigmund & 46 & Calman & Cernăuți \\
\hline 104 & Held Paulette & 4 & Sigmund & Cernăuți \\
\hline 105 & Hellenbrand Luise & 22 & Pascal & Bucharest \\
\hline 106 & Herscovici & 17 & Iosub & Vaslui \\
\hline 107 & Herscovici Samson & 17 & Marcu & Iași \\
\hline 108 & Herscovici Artenie & 20 & Maurici & Timișoara \\
\hline 109 & Hirsch Geza & 23 & Maurici & Târnăveni \\
\hline 110 & Hirschora Elias & 11 & Adolf & Bucharest \\
\hline 111 & Iosipovici Leon & 50 & Iancu & Vaslui \\
\hline 112 & Iosipovici Ana & 47 & Hirsch & Vaslui \\
\hline 113 & Iosef Noiman & 19 & Francisc & Timișoara \\
\hline 114 & Israel Simon & 25 & Haim & Budapest \\
\hline 115 & Israel Simon & 25 & Israle & Budapest \\
\hline 116 & Izbicki Avram & 29 & Zaev & Budapest \\
\hline
\end{tabular}




\section{Philosophy International Journal}

\begin{tabular}{|c|c|c|c|c|}
\hline 117 & Itzic Ernestina & 24 & Hersch & Iași \\
\hline 118 & Katz Aron & 53 & Eoch & Galați \\
\hline 119 & Katz Ludmila & 51 & Haim Wolf & Galați \\
\hline 120 & Kaufman Magda & 22 & Iosif & Timișoara \\
\hline 121 & Kaufman Mendel & 32 & Michael & Iași \\
\hline 122 & Kaufman Caesar & 26 & Mendel & Iași \\
\hline 123 & Koller Vera & 17 & Jacov & Ladois \\
\hline 124 & Koru Henrietta & 31 & Avram & Bucharest \\
\hline 125 & Klingher Suzana & 17 & Valter & Timișoara \\
\hline 126 & Kora Carol & 21 & Solomon & Cernăuți \\
\hline 127 & Kraft Maximilian & 41 & Jacob & Cernăuți \\
\hline 128 & Kraft Felicia & 11 & Maximilian & Cernăuți \\
\hline 129 & Kula Max Dr. & 45 & Berl & Cernăuți \\
\hline 130 & Kula Sara & 30 & Isac & Cernăuți \\
\hline 131 & Lang Josub & 25 & Bercu & Bucharest \\
\hline 132 & Laufer Israel & 33 & Feivel & Gura-Humorului \\
\hline 133 & Laufer Elsa & 30 & Leon & Gura-Humorului \\
\hline 134 & Landau Isac & 38 & Josef & Cernăuți \\
\hline 135 & Landau Rebecca & 36 & Ario Leila & Cernăuți \\
\hline 136 & Leb Andrei & 26 & Marcus & Turda \\
\hline 137 & Lederkramer Ithac & 30 & Jacov & Lemberg \\
\hline 138 & Leib Solomon & 22 & Simon & Iași \\
\hline 139 & Leib Haim & 20 & Hers & Iași \\
\hline 140 & Leiba Simon & 21 & Leiba & Iași \\
\hline 141 & Leibovici Rene Tuca & 17 & Carol & Bucharest \\
\hline 142 & Lene Ghizela & 30 & Naftali & Bucharest \\
\hline 143 & Lenz Mosche & 33 & Isac & Moldovița \\
\hline 144 & Lenz Fany & 24 & Iosef & Moldovița \\
\hline 145 & Lerner Iosef & 30 & Eugen & Bucharest \\
\hline 146 & Lindenberg Marian & 17 & Valcea & Bucharest \\
\hline 147 & Littman Sofica & 28 & David & Bucharest \\
\hline 148 & Lobel Leib Lea & 21 & Herscu & Iași \\
\hline 149 & Lupu Haim & 20 & Leiba & Iași \\
\hline 150 & Lupu Hadassa & 17 & Leiba & Iași \\
\hline 151 & Lupu Ada & 16 & Leiba & Iaşi \\
\hline 152 & Lupu Bela & 20 & Zisu & Focșani \\
\hline 153 & Lustig Emil & 49 & Isac & Zakopanc \\
\hline 154 & Mandl Adalbert & 23 & Dezideriu & Arad \\
\hline 155 & Mark Iosef & 18 & Avraham & Craiova \\
\hline 156 & Mayer Isidor & 19 & Simon & Bucharest \\
\hline
\end{tabular}




\section{Philosophy International Journal}

\begin{tabular}{|c|c|c|c|c|}
\hline 157 & Meickler Bania & 21 & Isac & Cernăuți \\
\hline 158 & Meier Natan & 20 & Elias & Fălticeni \\
\hline 159 & Mendelovici Saie & 24 & Moise & Tg. Neamt \\
\hline 160 & Mizrachi Solomon & 36 & Avram & Bucharest \\
\hline 161 & Mizrachi Odeta & 28 & Max & Bucharest \\
\hline 162 & Moscovici Max & 16 & Marcu & Iași \\
\hline 163 & Mulder Sara & 25 & Maier & Budapest \\
\hline 164 & Naftule Liviu & 19 & Iancu & Iași \\
\hline 165 & Nemetz Haim Meir & 19 & Herman & Timișoara \\
\hline 166 & Neufeld Gabriel & 47 & Isac & Storojineț \\
\hline 167 & Neufeld Eva & 39 & Peisach & Storojineț \\
\hline 168 & Neufeld Isac & 18 & Gabriel & Storojineț \\
\hline 169 & Neufeld Rubin & 6 & Gabriel & Storojineț \\
\hline 170 & Neuman Mauricius & 23 & Haschel & Timișoara \\
\hline 171 & Niederberg Raschela-Erika & 18 & Burach & Bucharest \\
\hline 172 & Nuta Nata & 19 & Avram & Iași \\
\hline 173 & Offenberg Marcel & 1 & Mendel & Bucharest \\
\hline 174 & Ordentlich Hanna & 35 & Avram & Timișoara \\
\hline 175 & Ordentlich Juval & 4 & Adolf & Timișoara \\
\hline 176 & Pomarleanu Lupu & 50 & Iancu & Iași \\
\hline 177 & Pomarleanu Sofia & 46 & Marcu & Iași \\
\hline 178 & Preminger Eugenia & 34 & Ozias & Cernăuți \\
\hline 179 & Rabinovici Herman & 21 & Josef & Bârlad \\
\hline 180 & Rabbun Jetti & 25 & Manase & Iași \\
\hline 181 & Rabinsohn Solomon & 46 & Zwy & Bucharest \\
\hline 182 & Rabinsohn Dina & 42 & Haim & Bucharest \\
\hline 183 & Rabinsohn Zipora & 14 & Solomon & Bucharest \\
\hline 184 & Rabinsohn Isa & 12 & Solomon & Bucharest \\
\hline 185 & Rabinovici Josef & 25 & Rubin & Bucharest \\
\hline 186 & Rabinovici Duta & 16 & Avram & Iași \\
\hline 187 & Rabinovitz Surica & 22 & Zalman & Vaslui \\
\hline 188 & Rachmut Haim Josef & 31 & Osias & Botoșani \\
\hline 189 & Rachmut Feiga & 23 & Mosche & Botoșani \\
\hline 190 & Reiser David & 21 & Menachem & Iași \\
\hline 191 & Reth Mauriciu Leon & 52 & Mozes & Radăuți \\
\hline 192 & Rim Esther & 43 & Burach & Cernăuți \\
\hline 193 & Rim Zigmund & 22 & Mozes & Cernăuți \\
\hline 194 & Ritter Adolf & 21 & Mauriciu & Târnăveni \\
\hline 195 & Riveles David & 48 & Sabse & Chișinău \\
\hline 196 & Riveles Tila & 37 & Salman & Chișinău \\
\hline
\end{tabular}




\begin{tabular}{|c|c|c|c|c|}
\hline 197 & Riveles Mary & 18 & David & Chișinău \\
\hline 198 & Rivensohn Sura & 61 & Fischel & Cernăuți \\
\hline 199 & Roll Manciu & 38 & Solomon & Vatra-Dornei \\
\hline 200 & Roll Gusta & 34 & Moritz & Vatra-Dornei \\
\hline 201 & Romer Alexandru & 22 & Cheiza & Timișoara \\
\hline 202 & Rosanblum Michael & 46 & Avram Iancu & Bucharest \\
\hline 203 & Rosanblum Rosalia & 40 & Julius & Bucharest \\
\hline 204 & Rosanblum Alexandru & 18 & Michael & Bucharest \\
\hline 205 & Rosenstein Natan & 22 & Strul & Bucharest \\
\hline 206 & Rosenthal Leib & 17 & Josef & Iași \\
\hline 207 & Rosentzweig Jacquest & 36 & Mordechai & Iași \\
\hline 208 & Rosentzweig Rivca & 38 & Iohosua & Iași \\
\hline 209 & Rosenzweig Lea & 68 & Josub & Jani \\
\hline 210 & Rosenzweig Rebeca & 33 & Mordechai & Jani \\
\hline 211 & Rotenstein Loti & 16 & Manase & Iași \\
\hline 212 & Roth Fani & 21 & Rudolf & Mediaș \\
\hline 213 & Rubinstein Ferdinand & 22 & Isidor & Timișoara \\
\hline 214 & Rudberg Martin & 36 & Elias & Bucharest \\
\hline 215 & Jvette & 21 & Michael & Bucharest \\
\hline 216 & Safran Josef Dr. Rav. & 33 & Bezakel & Bacău \\
\hline 217 & Safran Esther & 33 & Jacob Zeev & Bacău \\
\hline 218 & Safran Miriam & 5 & Josef & Bacău \\
\hline 219 & Saie Lieba & 22 & Strul Moise & Iași \\
\hline 220 & Saler Valeriu & 11 & Peisic & Bacău \\
\hline 221 & Samuel Rudolf & 37 & Isac & Bucharest \\
\hline 223 & Samuel Laura & 31 & Leon & Bucharest \\
\hline 224 & Samuelli Corneliu & 20 & Bernard & Bucharest \\
\hline 225 & Schaier Mina & 18 & Pincas & Bucharest \\
\hline 226 & Scheinberg Mandel & 65 & Zvi & Bucharest \\
\hline 227 & Scherer Sami & 29 & Moses & Cernăuți \\
\hline 228 & Schneier Paulina & 50 & Iosef & Iași \\
\hline 229 & Schoichet Isac & 54 & Zimon & Cernăuți \\
\hline 230 & Schoichet Sura & 44 & Joel & Cernăuți \\
\hline 231 & Schonbrun Andrei & 21 & Avram & Timișoara \\
\hline 232 & Schvadron Jacob & 36 & Avram & Bucharest \\
\hline 233 & Schvadron Hana & 31 & Dov & Bucharest \\
\hline 234 & Schvartz Avigdor & 22 & Sigismund & Târnăveni \\
\hline 235 & Schwartzmann Grisa & 22 & Haim & Bucharest \\
\hline 236 & Scupper Rosalia & 52 & Haim & Chișinău \\
\hline 237 & Scupper Anatolie & 31 & Isac & Chișinău \\
\hline
\end{tabular}




\section{Philosophy International Journal}

\begin{tabular}{|c|c|c|c|c|}
\hline 238 & Scupper Susana & 22 & Isac & Chișinău \\
\hline 239 & Sebel Jtzchak & 54 & Chisinau & Chișinău \\
\hline 240 & Sebel Rachel & 54 & Isac & Chișinău \\
\hline 241 & Sebel Avram & 15 & Isac & Chișinău \\
\hline 242 & Secher Leon & 39 & Aron & Bucharest \\
\hline 243 & Secher Tamara & 37 & Gherson & Bucharest \\
\hline 244 & Seckler Alfred & 38 & Heinrich & Cernăuți \\
\hline 245 & Seckler Cosma & 34 & Berl & Cernăuți \\
\hline 246 & Seckler Gabriella & 7 & Alfred & Cernăuți \\
\hline 247 & Seidler Herman & 57 & Solomon & Cernăuți \\
\hline 248 & Seidler Rebeca & 54 & Moritz & Cernăuți \\
\hline 249 & Seidler Camilo & 31 & Herman & Cernăuți \\
\hline 250 & Seidler Blima & 27 & Bercu & Cernăuți \\
\hline 251 & Seifer Achiva & 57 & Ascher & Bucharest \\
\hline 252 & Seifer Sara & 57 & Mosche & Bucharest \\
\hline 253 & Sepcaru Rosalia & 22 & Herman & Bucharest \\
\hline 254 & Sestopalli Fimi & 33 & Emanuel & Bucharest \\
\hline 255 & Sestopalli Elisabeta & 29 & Adalbert & Bucharest \\
\hline 256 & Siegermann Sorica & 20 & Saul & Bucharest \\
\hline 257 & Singer Eugen Jose & 30 & Leon & Bucharest \\
\hline 258 & Singer Frida Mancu & 29 & Meir & Bucharest \\
\hline 259 & Sneier Scharlotte & 18 & Froim & Iași \\
\hline 260 & Sobelman Beila & 26 & Haskal & Iași \\
\hline 261 & Solomon Simon & 38 & Herman & Bucharest \\
\hline 262 & Solomon Jeny & 34 & Moise & Bucharest \\
\hline 263 & Solomon Valentin & 3 & Simon & Bucharest \\
\hline 264 & Solomon Eva & 23 & Ignatz & Budapest \\
\hline 265 & Solomon Petre & 21 & Elias & Bucharest \\
\hline 266 & Solomon Zurica & 17 & Zisu & Bacău \\
\hline 267 & Sommer Sonia & 3 & Haskel & Cernăuți \\
\hline 268 & Sommer Jennetta & 20 & Mozes & Cernăuți \\
\hline 269 & Stauber Israel & 20 & Armin & Timișoara \\
\hline 270 & Steinmetz Inhosua & 33 & Saie Leib & Cernăuți \\
\hline 271 & Stern Tiberiu & 21 & Ferdinand & Orăștie \\
\hline 272 & Spieler Israel Smuel & 30 & Iosef & Krakau \\
\hline 273 & Stisber Karl & 17 & Smil & Bucharest \\
\hline 274 & Teitler Heinrich & 29 & Avram & Cernăuți \\
\hline 275 & Teitler Gizea & 23 & Lazar & Cernăuți \\
\hline 276 & Tenenbaum Aron & 19 & Iosef & Bucharest \\
\hline 277 & Theller Heinrich & 47 & Tobias & Bucharest \\
\hline
\end{tabular}




\section{Philosophy International Journal}

\begin{tabular}{|c|c|c|c|c|}
\hline 278 & Theller Mina & 40 & Simon & Bucharest \\
\hline 279 & Theller Rudolf & 44 & Tobias & Bucharest \\
\hline 280 & Theller Ella & 41 & Jaucu & Bucharest \\
\hline 281 & Theller Rene & 16 & Rudolf & Bucharest \\
\hline 282 & Tutman Inzer & 46 & Jacob Meir & Cernăuți \\
\hline 283 & Tutman Sabina & 44 & Marcus & Cernăuți \\
\hline 284 & Tutman Roza & 20 & Lazar & Cernăuți \\
\hline 285 & Vatarescu Jean & 22 & Emanuel & Bucharest \\
\hline 286 & Vilenco Marcel & 37 & Mosche & Cernăuți \\
\hline 287 & Vilenco Etillie & 36 & Hersch & Cernăuți \\
\hline 288 & Vilenco Martin & 7 & Marcel & Cernăuți \\
\hline 289 & Vulkan Natan & 23 & Dov & Budapest \\
\hline 290 & Wagner Max & 46 & Samuel & Cernăuți \\
\hline 291 & Wagner Dora & 42 & Ohers & Cernăuți \\
\hline 292 & Wainer Bernard & 48 & Burach & Cernăuți \\
\hline 293 & Wainer Roza & 39 & Isac & Cernăuți \\
\hline 294 & Wainer Nelly & 13 & Bernard & Cernăuți \\
\hline 295 & Wainer Benedict & 7 & Bernard & Cernăuți \\
\hline 296 & Wasilkovaky Alex & 29 & Israel & Lemberg \\
\hline 297 & Watter Adolf & 44 & Mayer & Cernăuți \\
\hline 298 & Watter Clara & 34 & Josef & Cernăuți \\
\hline 299 & Weinbach Silvia & 21 & Berthold & Budapest \\
\hline 300 & Weinberger Sava & 18 & Isidor & Arad \\
\hline 301 & Wendkert Adolf & 45 & Smil & Bucharest \\
\hline 302 & Weintraub Aron & 32 & Mendel & Milnitzo \\
\hline 303 & Weiss Eva & 19 & Albert & Turda \\
\hline 304 & Weiss Lia & 17 & Josef & Cluj \\
\hline 305 & Weiss Armand & 21 & Bella & Budapest \\
\hline 306 & Weissman Sulem & 27 & Falik & Focșani \\
\hline 307 & Weissman Sura Zel & 21 & Falik & Focșani \\
\hline 308 & Weissman Manase & 21 & Saie & Tg. Neamț \\
\hline 309 & Wertberger Rachmil & 21 & Isack Arie & Arad \\
\hline 310 & Wieder Hermina & 17 & Lazar & Bucharest \\
\hline 311 & Winic Lucie & 34 & Goga & Bucharest \\
\hline 312 & Wurnbrand Max & 42 & Heinrich & Bucharest \\
\hline 313 & Wurnbrand Frima & 40 & Moise & Bucharest \\
\hline
\end{tabular}

\section{Conclusion}

The Alia movement in Romania during the Second World War was one of the pages of heroism of emigration to Israel. The movement saved thousands of Jews but also meant the sacrifice of 1,200 Jews, who disappeared in the sea ${ }^{6}$.

6 Arie Steinberg, Aspecte internaționale ale emigrării evreiești din România și prin România 1938-1947,Haifa, 1984, pp. 363-373. 


\section{Acknowledgements}

This work is supported by project POCU 125040, entitled "Development of the tertiary university education to support the economic growth - PROGRESSIO", co-financed by the European Social Fund under the Human Capital Operational Program 2014-2020

\section{References}

1. ACIER, fond III, dosar 361, pp: 120-124.

2. Barles, H (1975) Acțiunile de salvare din perioada
Holocaustului. Tel Aviv.

3. Geller I (1991) Tragedia scufundării vasului Mefkure, august 1944. In: Holocaust, 19th (Vol.). Tel Aviv.

4. Koslinski N, Stănescu R (1998) Marina Română în al doilea război mondial (1939-1945). 3rd (Vol.) (19441945), București, Editura Modelism Internațional.

5. Naor M (1981) Emigrarea 1934-1948. Tel Aviv.

6. Steinberg A (1984) Aspecte internaționale ale emigrării evreiești din România și prin România 1938-1947. Haifa. 